\title{
Mathematical modeling of three - dimensional genetic regulatory networks using logistic and Gompertz functions
}

\author{
INNA SAMUILIK ${ }^{1}$, FELIX SADYRBAEV ${ }^{1,2}$, DIANA OGORELOVA ${ }^{1}$ \\ ${ }^{1}$ Department of Natural Sciences and Mathematics \\ Daugavpils University \\ Parades street 1 \\ LATVIA \\ ${ }^{2}$ Institute of Mathematics and Computer science \\ University of Latvia \\ Rainis boulevard 29 \\ LATVIA
}

\begin{abstract}
Mathematical modeling is a method of cognition of the surrounding world in which the description of the object is carried out in the language of mathematics, and the study of the model is performed using certain mathematical methods. Mathematical models based on ordinary differential equations (ODE) are used in the study of networks of different kinds, including the study of genetic regulatory networks (GRN). The use of ODE makes it possible to predict the evolution of GRN in time. Nonlinearity in these models is included in the form of a sigmoidal function. There are many of them, and in the literature, there are models that use different sigmoidal functions. The article discusses the models that use the logistic function and Gompertz function. The comparison of the results, related to three-dimensional networks, has been made. The text is accompanied by examples and illustrations.
\end{abstract}

Key-Words: gene regulatory network, Gompertz function, logistic function, periodic solutions

Received: March 28, 2021. Revised: January 16, 2022. Accepted: February 5, 2022. Published: February 23, 2022.

\section{Introduction}

The main problem in mathematical modeling of a dynamic system is to develop a model and then to determine dependencies and coefficients in the equations used in developing the model. For complex dynamic systems, the determination of the coefficients and dependencies in the model is a nontrivial task.

In nonlinear models, we consider cycles, stable and unstable regimes [11], a strange attractor and chaos [1]. Cycles are regular impacts on the economic mechanism with a period of one year (autumn harvest, increased heating costs in the winter season). Chaos, in socio-economic systems and biological communities can be interpreted as a natural form of competition. Artificial elimination of chaos (in mechanics - due to a large dissipation of energy, in the economy - due to excessive regulation, high taxation, in society - due to legislative restrictions) leads to the elimination of complex dynamic regimes and the transition to simple solutions, degradation of the system. In mechanics, these are the usual simplest periodic fluctuations, in economics - a situation of stagnation. During the transition from chaos to orderliness or after the loss of stability of the previous regime, new stable non-trivial solutions arise in mechanics as well as inprofitable directions in the economy.

Consider the general form of writing the $n$ dimensional dynamical system, that is expected to model a genetic regulatory network,

$\left\{\begin{array}{l}x^{\prime}{ }_{1}=\frac{1}{1+e^{-\mu_{1}\left(w_{11} x_{1}+w_{12} x_{2}+\cdots+w_{1} x_{\mathrm{n}}-\theta_{1}\right)}}-v_{1} x_{1}, \\ x^{\prime}{ }_{n}=\frac{1}{1+e^{-\mu_{\mathrm{n}}\left(w_{\mathrm{n} 1} x_{1}+w_{\mathrm{n} 2} x_{2}+\cdots+w_{\mathrm{nn}} x_{\mathrm{n}}-\theta_{\mathrm{n}}\right)}}-v_{\mathrm{n}} x_{\mathrm{n}},\end{array}\right.$ or 


$$
\left\{\begin{array}{l}
x_{1}^{\prime}=e^{-e^{-\mu_{1}\left(w_{11} x_{1}+w_{12} x_{2}+\cdots+w_{1 \mathrm{n}} x_{\mathrm{n}}-\theta_{1}\right)}}-v_{1} x_{1}, \\
\cdots \\
x_{n}^{\prime}=e^{-e^{-\mu_{\mathrm{n}}\left(w_{\mathrm{n} 1} x_{1}+w_{\mathrm{n} 2} x_{2}+\cdots+w_{\mathrm{nn}} x_{\mathrm{n}}-\theta_{\mathrm{n}}\right)}}-v_{\mathrm{n}} x_{\mathrm{n}}
\end{array}\right.
$$

where $\mu_{i}>0, \theta_{i}$ and $v_{i}>0$ are parameters, and $w_{i j}$ are elements of the $n \times n$ regulatory matrix $W$. The parameters of the GRN have the following biological interpretations:

$v_{i}$ - the rate of degradation of the $i$-th gene expression product;

$w_{i j}-$ the connection weight or strength of control of gene $j$ on gene $i$. Positive values of $w_{i j}$ indicate activating influences while negative values define repressing influences;

$\theta_{i}$-the influence of external input on gene $i$, which modulates the gene's sensitivity of response to activating or repressing influences. [13]

The sigmoidal functions $f(z)=\frac{1}{1+e^{-\mu z}} \quad$ and $f(z)=e^{-e^{-\mu(z-\theta)}}$ are used in (1).

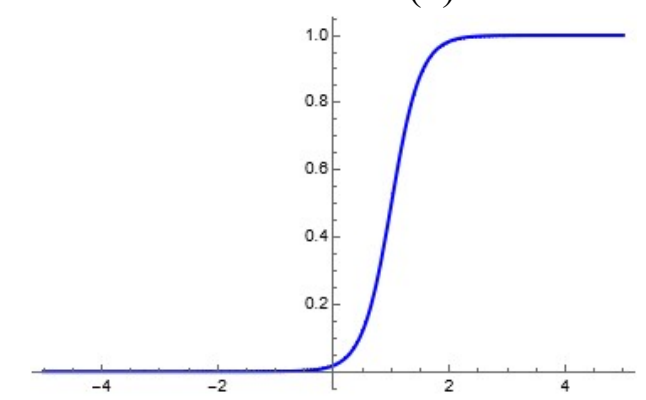

Figure 1.Logistic function.

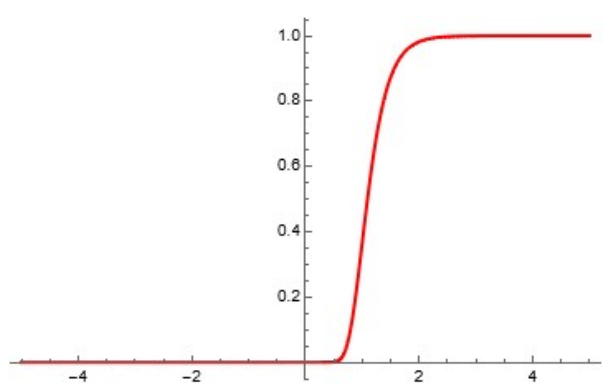

Figure 2. Gompertz function.

Sigmoidal functions are monotonically increasing from zero to unity and have a single inflection point. They are many, but the above functions suit well for the analysis and visualizations. A set of coefficients $w_{i j}$ form the so-called regulatory matrix

$$
W=\left(\begin{array}{ccc}
w_{11} & \ldots & w_{1 n} \\
\ldots & \ldots & \ldots \\
w_{n 1} & \ldots & w_{n n}
\end{array}\right)
$$

\section{Three-element GRN}

Consider the three-dimensional system for Logistic function

$\left\{\begin{array}{l}x_{1}^{\prime}=\frac{1}{1+e^{-\mu_{1}\left(w_{11} x_{1}+w_{12} x_{2}+w_{13} x_{3}-\theta_{1}\right)}-v_{1} x_{1},} \\ x_{2}^{\prime}=\frac{1}{1+e^{-\mu_{2}\left(w_{21} x_{1}+w_{22} x_{2}+w_{23} x_{3}-\theta_{2}\right)}-v_{2} x_{2},(3)} \\ x_{3}^{\prime}=\frac{1}{1+e^{-\mu_{3}\left(w_{31} x_{1}+w_{32} x_{2}+w_{33} x_{3}-\theta_{3}\right)}}-v_{3} x_{3},\end{array}\right.$

and for Gompertz function

$\left\{\begin{array}{l}x_{1}^{\prime}=e^{-e^{-\mu_{1}\left(w_{11} x_{1}+w_{12} x_{2}+w_{13} x_{3}-\theta_{1}\right)}-v_{1} x_{1},} \\ x_{2}^{\prime}=e^{-e^{-\mu_{2}\left(w_{21} x_{1}+w_{22} x_{2}+w_{23} x_{3}-\theta_{2}\right)}-v_{2} x_{2},} \\ x_{3}^{\prime}=e^{-e^{-\mu_{3}\left(w_{31} x_{1}+w_{32} x_{2}+w_{33} x_{3}-\theta_{3}\right)}-v_{3} x_{3} .}\end{array}\right.$

The nullclines for the system (3) are defined by the relations

$$
\left\{\begin{array}{l}
x_{1}=\frac{1}{v_{1}} \frac{1}{1+e^{-\mu_{1}\left(w_{11} x_{1}+w_{12} x_{2}+w_{13} x_{3}-\theta_{1}\right)}} \\
x_{2}=\frac{1}{v_{2}} \frac{1}{1+e^{-\mu_{2}\left(w_{21} x_{1}+w_{22} x_{2}+w_{23} x_{3}-\theta_{2}\right)}} \\
x_{3}=\frac{1}{v_{3}} \frac{1}{1+e^{-\mu_{3}\left(w_{31} x_{1}+w_{32} x_{2}+w_{33} x_{3}-\theta_{3}\right)}}
\end{array}\right.
$$

For the system (4) the nullclines are defined by the relations

$$
\left\{\begin{array}{l}
x_{1}=\frac{1}{v_{1}} e^{-e^{-\mu_{1}\left(w_{11} x_{1}+w_{12} x_{2}+w_{13} x_{3}-\theta_{1}\right)}} \\
x_{2}=\frac{1}{v_{2}} e^{-e^{-\mu_{2}\left(w_{21} x_{1}+w_{22} x_{2}+w_{23} x_{3}-\theta_{2}\right)}} \\
x_{3}=\frac{1}{v_{3}} e^{-e^{-\mu_{3}\left(w_{31} x_{1}+w_{32} x_{2}+w_{33} x_{3}-\theta_{3}\right)}}
\end{array}\right.
$$

\subsection{Logistic function}

Case 1. Stable limit cycles can exist in systems of the form (3). Consider the system (3) with the matrix

$$
W=\left(\begin{array}{ccc}
0 & 1 & 0 \\
-1 & 1 & 0 \\
1 & 1 & 0.01
\end{array}\right)(7)
$$

and $\mu_{1}=\mu_{3}=5, \mu_{2}=15 ; \quad v_{1}=v_{2}=v_{3}=1$; $\theta_{1}=0.5, \theta_{2}=0.04, \theta_{3}=-0.5$. Three nullclines 
WSEAS TRANSACTIONS on SYSTEMS and CONTROL DOI: 10.37394/23203.2022.17.12 are located as shown in Figure 3. Computations and graphical results are performed using Wolfram Mathematica.

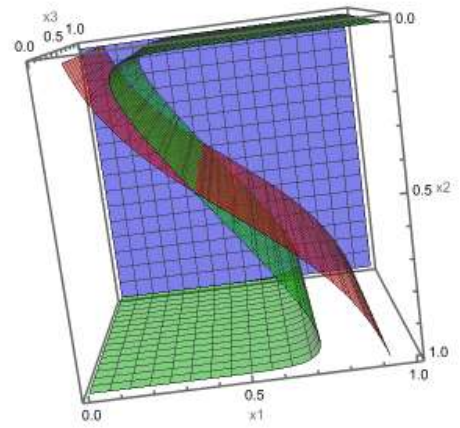

Figure 3. Nullclines $\left(x_{1}-\right.$ red, $x_{2}-$ green, $x_{3}-$ blue $)$

There is one critical point $p_{1}$ : (0.4018; 0.4204; 0.9987). Linearization around this point provides us with the characteristic numbers $\lambda$ given in Table 1 .

Table 1 . The characteristic numbers $\lambda$

\begin{tabular}{|c|c|c|c|}
\hline- & $\lambda_{1}$ & $\lambda_{2}$ & $\lambda_{3}$ \\
\hline$p_{1}$ & -0.9999 & $0.8275-1.0261 i$ & $0.8275+1.0261 i$ \\
\hline
\end{tabular}

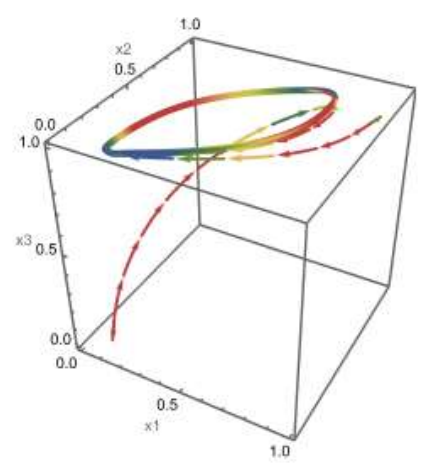

Figure 4. Periodic solutions

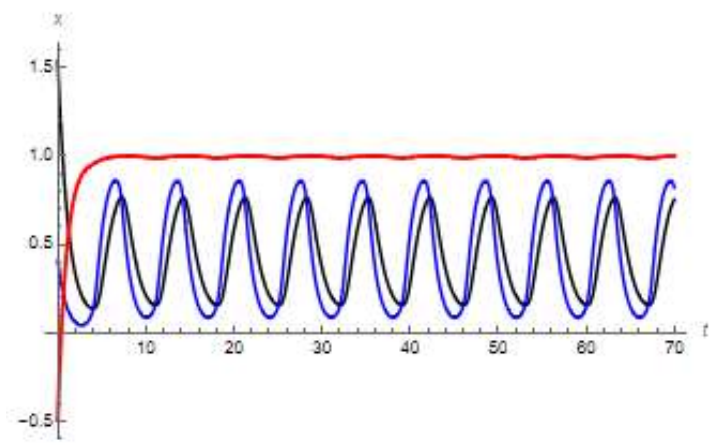

Figure 5. The graphs of $x_{i}(t), i=1,2,3$
Case 2. Consider the system (3) with the matrix

$$
W=\left(\begin{array}{ccc}
1.2 & 0 & 2 \\
0.5 & 1 & -0.5 \\
-2 & 0.01 & 1
\end{array}\right)(8)
$$

and $\mu_{1}=\mu_{3}=5, \mu_{2}=15 ; \quad v_{1}=v_{2}=v_{3}=1$ $\theta_{1}=1.0, \theta_{2}=0.4, \theta_{3}=-0.5$. Three nullclines are located as shown in Figure 6.

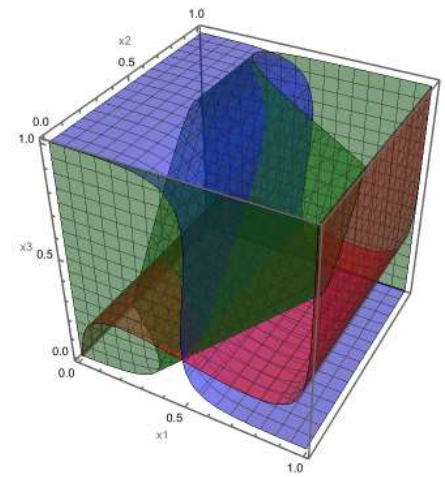

Figure 6. Nullclines $\left(x_{1}-\right.$ red, $x_{2}-$ green, $x_{3}-$ blue $)$

There are three critical points $p_{1}, p_{2}$ and $p_{3}:(0.4885 ; 0.0342 ; 0.2023)$, (0.4890; 0.1297; 0.2022), (0.4935; 0.9999; 0.2013). Linearization around these points provides us with the characteristic numbers $\lambda$ given in Table 2 .

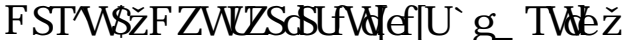

\begin{tabular}{|c|c|c|c|}
\hline- & $\lambda_{1}$ & $\lambda_{2}$ & $\lambda_{3}$ \\
\hline $\boldsymbol{p}_{1}$ & -0.5026 & $0.1522-1.9782 i$ & $0.1522+1.9782 i$ \\
\hline $\boldsymbol{p}_{2}$ & 0.6965 & $0.1511-1.9799 i$ & $0.1511+1.9799 i$ \\
\hline $\boldsymbol{p}_{3}$ & -0.9998 & $0.1518-1.9743 i$ & $0.1518+1.9743 i$ \\
\hline
\end{tabular}

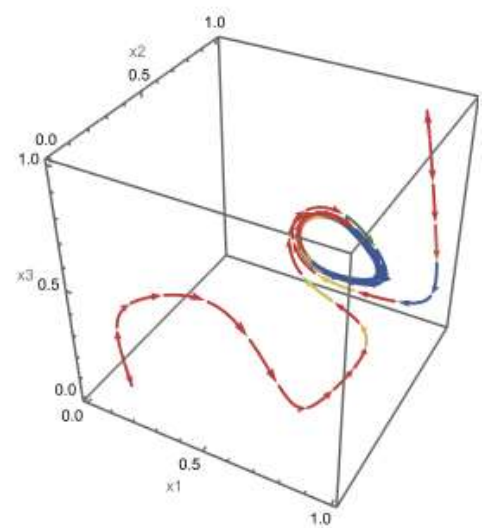

Figure 7. Periodic solutions 
WSEAS TRANSACTIONS on SYSTEMS and CONTROL DOI: 10.37394/23203.2022.17.12
Inna Samuilik, Felix Sadyrbaev, Diana Ogorelova

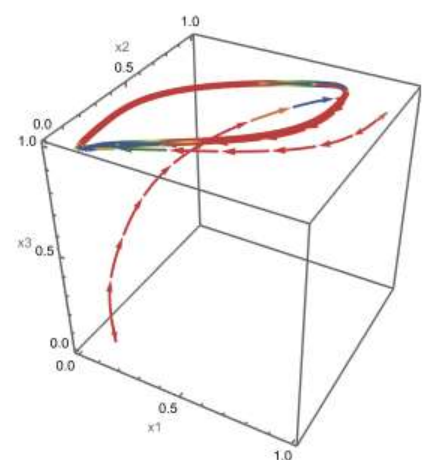

Figure 10. Periodic solutions

\subsection{Gompertz function}

Case 1. Stable limit cycles can exist in systems of the form (4). Consider the system (4) with the same matrix (7) and the same parameters $\mu, v$ and $\theta$. Three nullclines are located as shown in Figure 9. Computations and graphical results are performed using Wolfram Mathematica.

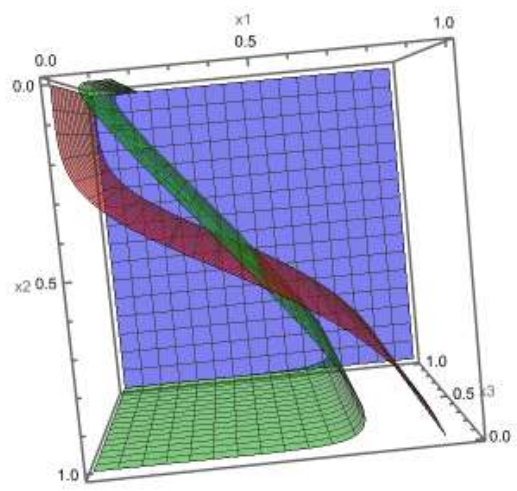

Figure 9. Nullclines $\left(x_{1}-\right.$ red, $x_{2}-$ green, $x_{3}-$ blue $)$

There is one critical point $p_{1}$ : (0.4894; $0.5672 ; 0.9996)$. Linearization around this point provides us with the characteristic numbers $\lambda$ given in Table 3 .

5 BOAMII I Fए BPDFSTLDON CFSTI

\begin{tabular}{|c|c|c|c|}
\hline- & $\lambda_{1}$ & $\lambda_{2}$ & $\lambda_{3}$ \\
\hline $\boldsymbol{p}_{1}$ & -0.9999 & $9.346-4.9497 i$ & $9.346+4.9497 i$ \\
\hline
\end{tabular}

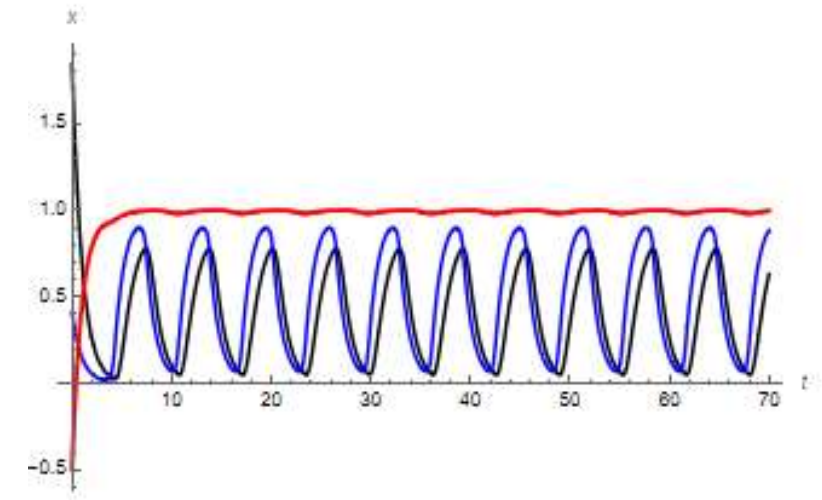

Figure 11 . The graphs of $x_{i}(t), i=1,2,3$

Case 2. Consider the system (4) with the same matrix (8) and the same parameters $\mu, v$ and $\theta$.

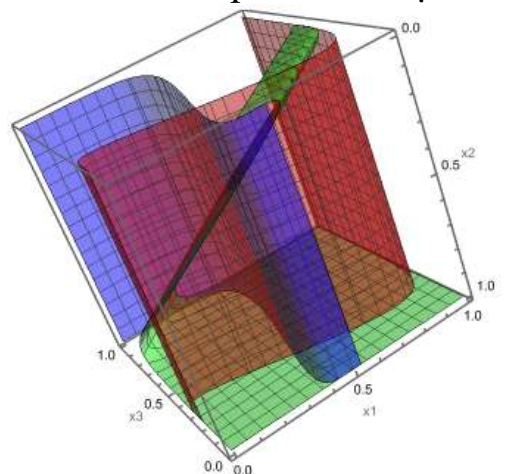

Figure 12. Nullclines $\left(x_{1}-\right.$ red, $x_{2}-$ green, $x_{3}-$ blue $)$

There are three critical points $p_{1}, p_{2}$ and $p_{3}$ : (0.4109; $0 ; 0.2652)$,

$(0.4124 ; 0.3169 ; 0.2647)$ and

(0.4156; 0.9999; 0.2637). Linearization around these points provides us with the characteristic numbers $\lambda$ given in Table 4 . 
WSEAS TRANSACTIONS on SYSTEMS and CONTROL DOI: $10.37394 / 23203.2022 .17 .12$
Table 4 . The characteristic numbers $\lambda$

\begin{tabular}{|c|c|c|c|}
\hline- & $\lambda_{1}$ & $\lambda_{2}$ & $\lambda_{3}$ \\
\hline $\boldsymbol{p}_{1}$ & -1 & $3.2096-5.75024 i$ & $3.2096+5.75024 i$ \\
\hline $\boldsymbol{p}_{2}$ & 12.3148 & $3.2286-5.73206 i$ & $3.2286+5.73206 i$ \\
\hline $\boldsymbol{p}_{3}$ & 14.0087 & $3.2278-5.80828 i$ & $3.2278+5.80828 i$ \\
\hline
\end{tabular}

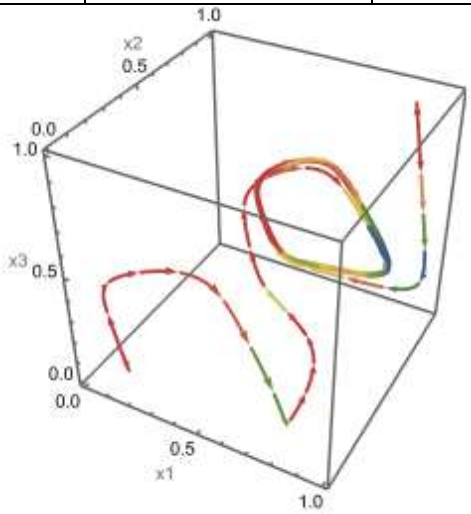

Figure 13. Periodic solutions

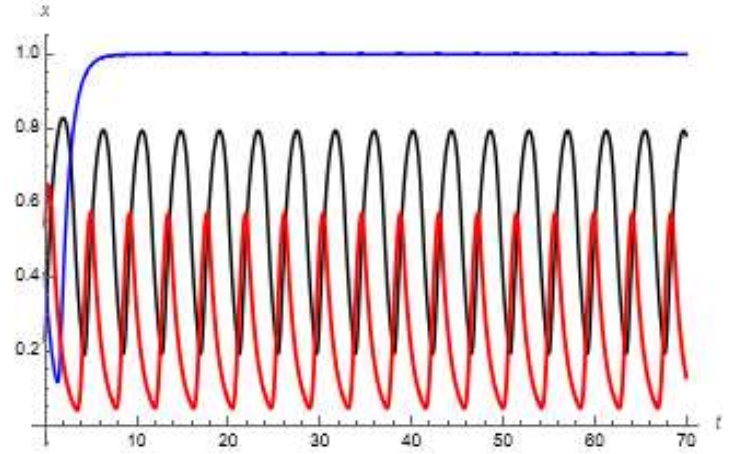

Figure 14 . The graphs of $x_{i}(t), i=1,2,3$

\section{More on 3D systems}

We will show now some differences when applying both functions. Consider system (3) with the following set of parameters: $v_{i}=1, \mu_{i}=10$, $\theta_{1}=1.5, \theta_{2}=-0.5, \theta_{3}=0.32$ and the regulatory matrix is

$$
W=\left(\begin{array}{ccc}
1 & 2 & 0 \\
-2 & 1 & 0 \\
0 & 0 & 1
\end{array}\right)
$$

This system is uncoupled. The 2-dimensional system with the matrix

$$
W_{1}=\left(\begin{array}{cc}
1 & 2 \\
-2 & 1
\end{array}\right)
$$

is known to have the periodic solution. The 1dimensional equation

$x_{3}^{\prime}=\frac{1}{1+e^{-\mu_{3}\left(x_{3}-\theta_{3}\right)}}-x_{3}$ has the nullcline which is a union of two points. These points are seen in the Figure 15.
Inna Samuilik, Felix Sadyrbaev, Diana Ogorelova

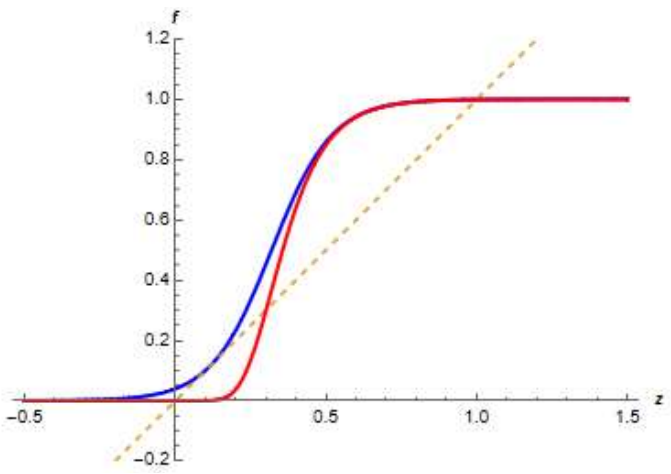

Figure 15. Logistic function (blue) and Gompertz function (red) for $\theta_{3}=0.32$.

The third nullcline for 3D-system with the logistic function consist of two planes of the form $x_{3}=p_{1}$ and $x_{3}=p_{2}$, where $p_{1,2}$ are roots of the equation $x_{3}=\frac{1}{1+e^{-\mu_{3}\left(x_{3}-\theta_{3}\right)}}$. They are two, one of them corresponds to the tangent point of the blue graph with the graph of the bisectrix. The 3D system has two periodic solutions that locate in the planes $x_{3}=p_{1}$ and $x_{3}=p_{2}$. Only the second one is attractive. Consider the 3D-system (4), where the Gompertz function is used in a model, and all the parameters are the same as above. Since the red curve has three points of intersection with the bisectrix, this system has three periodic solutions in the planes $x_{3}=q_{i}, i=1,2,3$, where the points $q_{1}, q_{2}, q_{3}$ are roots of the equation $x_{3}=$ $e^{-e^{-\mu_{3}\left(x_{3}-\theta_{3}\right)}}$. The first and the third periodic solutions are attractive. Do the same for both 3Dsystems where only the parameter $\theta_{3}$ is set for 0.15. The result is seen in Figure 16.

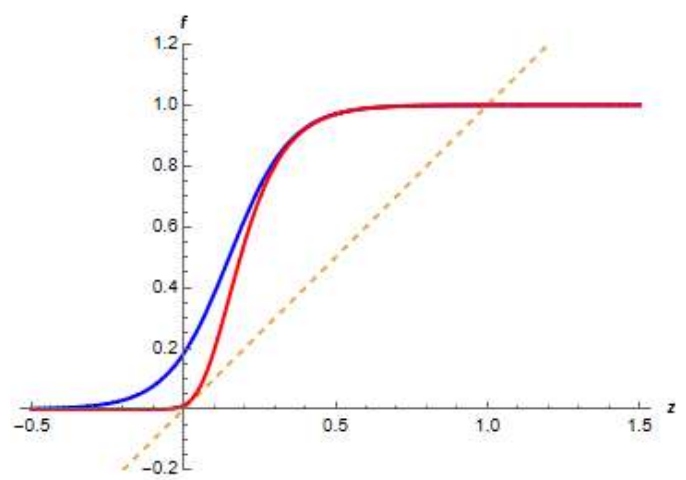

Figure 16. Logistic function (blue) and Gompertz function (red) for $\theta_{3}=0.15$. 
Now the first 3D system with the logistic function has one periodic solution, which is attractive. The second 3D system with Gompertz function has two periodic solutions corresponding to two roots of the equation $x_{3}=e^{-e^{-\mu_{3}\left(x_{3}-\theta_{3}\right)}}, \theta_{3}=0.15$.

We conclude that for the set of parameters listed above, where only $\theta_{3}$ varies, the following is true. For $0.15<\theta_{3}<0.32$ the first 3D system (3) has one periodic solution which is attractive. The second 3D-system (4) has three periodic solutions. Of them two periodic solutions are stable. The structure of phase spaces for the two systems is completely different despite of the fact that they use the same set of parameters.

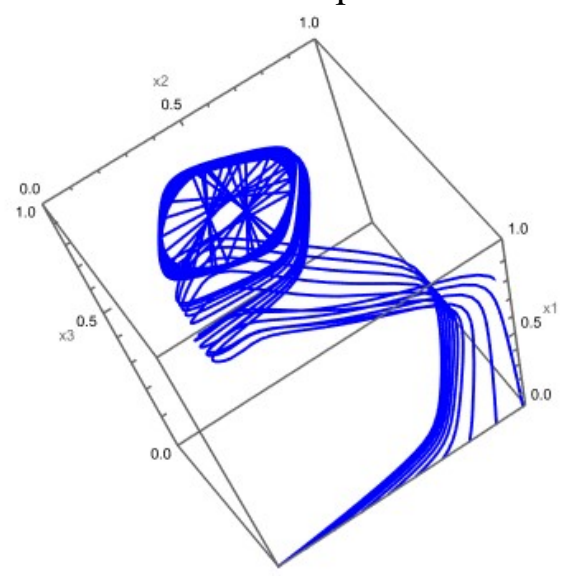

Figure 17. Logistic function, the attractive solution, $\theta_{3}=0.24$

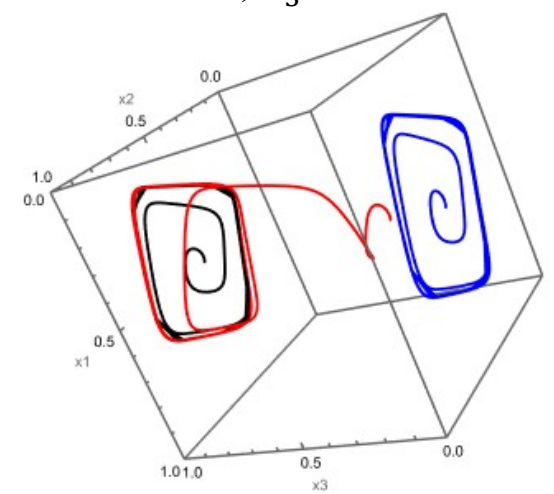

Figure 18. Gompertz function, three periodic solutions, $\theta_{3}=0.24$

\section{Conclusions}

The Gompertz function resembles a logistic function, both sigmoidal functions have a lot in common, but also a lot of differences. In the Gompertz function, growth deceleration does not occur as fast as its acceleration. Both functions are activation functions. In this paper we approach mathematical models of genetic networks. The same set of ordinary differential equations appear in models of telecommunication networks and neuronal networks. The systems of ODE are quasi-linear and nonlinearities are represented by sigmoidal functions. To which extent the results obtained can coincide and/or differ if different sigmoidal functions are used? We got partial answer to this question. We have studied two systems with the same sets of parameters (and they are many). The only difference was that in the first system the logistic function was used, while in the second one it was substituted by Gompertz function. Both functions are quite similar, but the second one uses double exponent and this makes it rapidly going to limits. Nevertheless, if both systems had a single critical point of the non-attractive nature, as expected, both had a stable periodic solution. For a different regulatory matrix (8) both systems had three critical points of non-attractive nature. Both systems had periodic solutions and behavior of solutions tending to this periodic one, is quite similar (Fig. 5 and Fig. 11). The section 3 is devoted to differences that can occur when applying both functions to the same model. The uncoupled systems were considered with the specific third nullcline, which could be in the form of one or three $x_{3}$-planes. Only one parameter, $\theta_{3}$, was allowed to vary. On a relatively long interval, $\theta_{3} \in(0.15,0.32)$, significant differencies between two systems were observed. The reason is that the third nullcline is defined by the equation of the form $x_{3}=f\left(x_{3}\right)$, where $f$ can be logistic or Gompertz function. For $\theta_{3}$ in the above interval these equations have different number of roots. This leads to substantial differencies in the strucrture of attractors in both systems. Stable periodic solutions serve as attractors in both systems, but their number (and location) is different. This may lead to 
misunderstanding in interpretation of behavior of modeled networks.

\section{Acknowledgements:}

ESF Project No. 8.2.2.0/20///003 "Strengthening of Professional Competence of Daugavpils University Academic Personnel of Strategic Specialization Branches 3rd Call”

\section{References}

[1]. A.Das, A.B.Roy, Pritha Das. Chaos in a three dimensional neural network. Applied Mathematical Modelling, 24(2000), 511-522.

[2]. A.Das, A.B.Roy, Pritha Das. (2002) Chaos in a Three Dimensional General Model of NeuralNetwork. International Journal of Bifurcation and Chaos, 12, 22712281. http://dx.doi.org/10.1142/S0218127402005820

[3] F. Sadyrbaev, I.Samuilik, V.Sengileyev, "On Modelling of Genetic Regulatory Networks," WSEAS Transactions on Electronics, vol. 12, pp. 73-80, 2021

[4] Le-Zhi Wang, Ri-Qi Su, Zi-Gang Huang,XiaoWang, WenXuWang,CelsoGrebogiandYingChengLai,Ageometrica lapproachto controland control abilityofnonlinear dynamicalnetworks.Nature Communications, Volume7, Article number:11323(2016), DOI:10.1038/ncomms11323

[5] H.D.Jong. Modeling and Simulation of GeneticRegulatorySystems: A Literature Review, J. Comput Biol. 2002;9(1):67-103, DOI: 10.1089/10665270252833208

[6] Y. Koizumi et al. Adaptive Virtual NetworkTopology Control Based on Attractor Selection.Journal of Lightwave Technology(ISSN: 0733-8724), Vol.28 (06/2010), Issue 11, pp.17201731DOI:10.1109/JLT.2010.2048412

[7] S. Atslega, F. Sadyrbaev, I. Samuilik. On Modelling Of Complex Networks. Engineering for Rural Development(ISSN 1691-5976), 2021, pp. 10091014.

[8] F. Sadyrbaev, I. Samuilik. Mathematical Modelling of Genetic Regulatory Networks. In: 2nd International Baku Conference on Scientific Research: The Book of Full Texts. Vol.1, Azerbaijan, Baku, 28-30 April, 2021. Baku: IKSAD GLOBAL Publications, 2021, pp.463-469. ISBN 978-60570554-6-0.

[9] F. Sadyrbaev, I. Samuilik. On the hierarchy of attractors in dynamical models of complex networks.19 Intern.Confer.Numer.Analys.andAppl.Mathematics,

Rhodes, Greece, 20-26 September 2021, To appear in AIP Conference Proceedings. https: //aip.scitation.org/journal/apc

[10]. N.Vijesh, S. Kumar Chakrabarti, J. Sreekumar. Modeling of gene regulatory networks: A review. J.
Biomedical Science and Engineering, 2013, 6, 223-231. http://dx.doi.org/10.4236/jbise.2013.62A027

[11] I. Samuilik, F. Sadyrbaev, "Mathematical Modelling of Leukemia Treatment", WSEAS Transactions on Computers, 20:274-281. DOI: 10.37394/23205.2021.20.30

[12] E. Ott, (2002). Chaos in Dynamical Systems (2nd ed.). Cambridge: Cambridge University Press. doi:10.1017/CBO9780511803260

[13] I. Samuilik, F. Sadyrbaev, "Modelling Three Dimensional Gene Regulatory Networks", WSEAS Transactions on Systems and Control 16:755-763. DOI: $\underline{10.37394 / 23203.2021 .16 .67}$

[14] J. C. Sprott. Elegant Chaos Algebraically Simple Chaotic Flows. World Scientific Publishing Company, 2010, 302 pages. https://doi.org/10.1142/7183

[15] S. Mukherjee, S. K. Palit, D. K. Bhattacharya. Is one dimensional Poincar map sufficient to describe the chaotic dynamics of a three dimensional system?. Applied Mathematics and Computation, Volume 219, Issue 23, 2013, Pages 11056-11064, ISSN 0096-3003. https://doi.org/10.1016/j.amc.2013.04.043

\section{Contribution of individual authors to the creation of a scientific article (ghost writing policy) \\ Allauthorshavecontributedequallyto creation nofthisarticle.}

\section{Sources of funding for research presented in a scientific article or scientific article itself}

ESF Project No. 8.2.2.0/20/I/003 "Strengthening of Professional Competence of Daugavpils University Academic Personnel of Strategic Specialization Branches 3rd Call"

\section{Creative Commons Attribution License}

\section{0}

\section{(Attribution 4.0 International, CC BY}

\section{0)}

This article is published under the terms of the CreativeCommons Attribution License 4.0 https://creativecommons.org/licenses/by/4.0/deed. en_US 